\title{
Guest Editorial: Special Issue on Parameterized and Exact Computation, Part II
}

\author{
Venkatesh Raman • Saket Saurabh
}

Received: 28 December 2012 / Accepted: 3 January 2013 / Published online: 8 January 2013

(C) Springer Science+Business Media New York 2013

The papers in this issue form the second part of the two parts special issue in the area of parameterized and exact exponential algorithms and complexity. While some of these are selected and extended papers from International Symposium on Parameterized and Exact Computation (IPEC) 2010, the rest were selected from the submissions received for our call for the special issue in the area.

The two parts reflect the volume of high quality submissions we received, which in turn reflects the high growth of the area in recent times.

While some of the papers in this volume supplement the ones in the first volume in the sub areas of kernelization and improved fixed-parameter algorithms for graph modification problems, most others complement them by focusing on problems in various application domains including Petri nets, evolutionary algorithms, topology, game theory, logic and biology.

The paper 'Small vertex cover makes Petri net coverability and boundedness easier' addresses two hard problems in the area of Petri nets. Coverability and boundedness problems in Petri nets are complete for the class of problems solvable in exponential space. However the paper shows that when the vertex cover number of the corresponding graph is a small parameter, these problems can be solved in space which is polynomial in the input size (with a super polynomial dependence on the vertex cover number). The paper 'Fixed-parameter evolutionary algorithms and the vertex cover problem' adopts a parameterized view to analyze the expected behavior of evolutionary algorithms. Using vertex cover as a case study, the paper explains how

V. Raman · S. Saurabh (区)

The Institute of Mathematical Sciences, Chennai, India

e-mail: saket@imsc.res.in

V. Raman

e-mail:vraman@imsc.res.in 
the behavior of general purpose search heuristics depends on structural properties of the problem.

The paper 'A tree traversal algorithm for decision problems in knot theory and 3-manifold topology' gives an efficient (in terms of time and space) exact exponential algorithm for an important problem in topology-normal surface enumeration. The paper 'Parameterized two-player Nash equilibrium' addresses a central and hard problem in game theory from the perspective of parameterized complexity. The problem of computing Nash equilibria in a two-player normal form game has been known to be a computationally hard problem. This paper identifies several (combination of) parameters in the input for which the problem becomes fixed-parameter tractable. The paper 'On the exact complexity of evaluating quantified $k-C N F$ ' addresses the exponential complexity of evaluating quantified boolean formula. In particular, it gives a lower bound for the complexity assuming strong exponential time hypothesis, and better upper bounds for several restricted cases. The paper 'Finding and counting vertex-colored subtrees' addresses a graph pattern-matching problem arising in the context of biological networks. The paper gives new improved fixed-parameter algorithms for Graph Motif problem and some of its variants.

The papers 'Proper interval vertex deletion' and 'Fast polynomial space algorithms using inclusion-exclusion' give improved fixed-parameter algorithms for some graph problems. The first one uses new structural results to give algorithms with improved running time to decide whether a given graph can be turned into a proper interval graph by deleting at most $k$ vertices. The second paper uses the principle of inclusion-exclusion to give polynomial space algorithm for several variants of spanning and Steiner tree problems.

In 'On the Grundy and b-chromatic numbers of a graph', the authors give hardness results and fixed-parameter algorithm for some variations of graph coloring problem. In 'On the (non-) existence of polynomial kernels for $P \_l$-free edge modification problems', the authors give positive results in the form of cubic vertex kernels, and negative results in the form of non-existence of polynomial sized kernels under complexity theoretic assumptions, for certain graph edge modification problems.

All the papers in the volume went through the rigorous refereeing process of Algorithmica. We thank all those who submitted papers in response to our call for papers, and the referees for their time and effort. Finally, we thank Ming-Yang Kao, editorin-chief of Algorithmica for his guidance throughout the production of this special issue. 